\title{
Relationship of Established Cardiovascular Risk Factors and Peripheral Biomarkers on Cognitive Function in Adults at Risk of Cognitive Deterioration
}

\author{
Michelle M.Y. Lai ${ }^{\mathrm{a}, \mathrm{b}, \mathrm{c}, *}$, Matthew J. Sharman ${ }^{\mathrm{d}}$, David J. Ames ${ }^{\mathrm{a}}$, Kathryn A. Ellis ${ }^{\mathrm{a}, \mathrm{k}}$, \\ Kay L. Cox ${ }^{\mathrm{e}}$, Graham Hepworth ${ }^{\mathrm{f}}$, Patricia Desmond ${ }^{\mathrm{g}}$, Elizabeth V. Cyarto ${ }^{\mathrm{a}, \mathrm{h}}$, \\ Ralph N. Martins ${ }^{\mathrm{i}, \mathrm{j}}$, Colin L. Masters ${ }^{\mathrm{k}}$ and Nicola T. Lautenschlager ${ }^{\mathrm{a}, 1, \mathrm{~m}}$ \\ ${ }^{a}$ Academic Unit for Psychiatry of Old Age, Department of Psychiatry, The University of Melbourne, \\ Melbourne, Australia \\ ${ }^{\mathrm{b}}$ South Metropolitan Health Service, Perth, Australia \\ ${ }^{\mathrm{c} C u r t i n}$ Medical School, Curtin University, Perth, Australia \\ ${ }^{\mathrm{d}}$ School of Health Sciences, University of Tasmania, Launceston, Tasmania, Australia \\ ${ }^{\mathrm{e}}$ Medical School, University of Western Australia, Perth, Australia \\ ${ }^{\mathrm{f}}$ Statistical Consulting Centre, The University of Melbourne, Melbourne, Australia \\ $\mathrm{g}^{\mathrm{g}}$ Department of Radiology, Royal Melbourne Hospital The University of Melbourne, Australia \\ ${ }^{\mathrm{h}}$ Bolton Clarke Research Institute, Melbourne, Australia \\ ${ }^{i}$ School of Medical Sciences, Edith Cowan University, Perth, Australia \\ ${ }^{\mathrm{j}}$ Department of Biomedical Sciences, Macquarie University, Sydney, NSW, Australia \\ ${ }^{\mathrm{k}}$ The Florey Institute, The University of Melbourne, Melbourne, Australia \\ ${ }^{1}$ WA Centre for Health \& Ageing, Medical School, University of Western Australia, Perth, Australia \\ ${ }^{\mathrm{m}}$ North Western Mental Health, Melbourne Health, Melbourne, Australia
}

Handling Associate Editor: Simon Laws

Accepted 24 December 2019

\begin{abstract}
.
Background: There is a paucity of information on the role of microvascular and inflammatory biomarkers in cognitive dysfunction.

Objective: This study sought to evaluate the relationships between established and a number of peripheral biomarkers on cognitive patterns in 108 older adults with memory complaints.

Methods: Participants in the AIBL Active study aged 60 years and older with at least one vascular risk factor and memory complaints completed a neuropsychological test battery and provided cross-sectional health data. Linear regression models adjusted for covariates examined associations between cognitive performance and a panel of vascular risk factors (Framingham cardiovascular scores, hs-CRP, homocysteine, fasting glucose, LDL-cholesterol) and peripheral biomarkers (TNF- $\alpha$, BDNF, VCAM-1, ICAM-1, PAI-1, CD40L).
\end{abstract}

\footnotetext{
${ }^{*}$ Correspondence to: Michelle M.Y. Lai, South Metropolitan Health Service, Locked Bag 100 Palmyra DC, WA 6961, Australia. Tel.: +61 088152 8205; E-mail: michelle.m.lai@ curtin.edu.au.
} 
Results: Higher fasting glucose and homocysteine levels were independent factors associated with poorer performance in Trail Making Test (TMT) B (adjusted $\beta=0.40 \pm 0.10$ and $0.43 \pm 0.09$, respectively). Increasing homocysteine levels were weakly associated with poorer global cognition and delayed recall (adjusted $\beta=0.23 \pm 0.10$ and $-0.20 \pm 0.10$ respectively). Increasing Framingham cardiovascular scores were related to poorer performance in TMT B $(\beta=0.42 \pm 0.19)$. There was early evidence of associations between increasing plasma TNF- $\alpha$ and poorer TMT B (adjusted $\beta=0.21 \pm 0.10$ ) and between increasing BDNF and better global cognition $(\beta=-0.20 \pm 0.09)$.

Conclusion: This study provides evidence to support the associations between vascular risk factors (Framingham scores, fasting glucose, and homocysteine) and poorer cognitive functions. Additionally, we measured several peripheral biomarkers to further investigate their associations with cognition. The relationship between TNF- $\alpha, B D N F$, and cognitive performance in various domains may offer new insights into potential mechanisms in vascular cognitive impairment.

Keywords: BDNF, biomarkers, cardiovascular disease, cognitive impairment, homocysteine, TNF- $\alpha$, vascular risk factors

Trial Registration: Australia New Zealand Clinical Trials Registry ACTRN126110006129

\section{INTRODUCTION}

Cognitive impairment commonly accompanies vascular pathology of the brain [1]. Vascular cognitive impairment refers to a spectrum of cognitive impairment associated with cerebrovascular disease, including vascular dementia, mixed Alzheimer's disease (AD) with a vascular component, and vascular cognitive impairment that does not meet dementia criteria [2]. Research studies indicate a complex interaction between cerebrovascular disease and AD [1]. Both conditions share common vascular risk factors and have overlapping ischemic and neurodegenerative pathology [3].

Early assessment of cardiovascular risk factors is important in clinical practice as overall cardiovascular health correlates with the risk for coronary heart disease, stroke, and vascular disease [4]. Midlife smoking, high cholesterol, hypertension, and diabetes were associated with $20-40 \%$ increased risk of dementia in a dose-dependent manner [5]. That the established cardiovascular risk factors are also risk factors for cognitive decline is increasingly recognized [2]. The cognitive pattern that reflects a vascular origin is predominantly subcortical with poorer performance in executive function and psychomotor slowing, but the cognitive expression of subcortical small vessel disease in individuals can be highly variable [1].

Microvascular endothelial dysfunction and inflammatory states are considered critical pathways linking vascular risk factors and dementia [6]. One example is the observed neuronal and endothelial injury promotion under the effect of homocysteine [7]. There is much speculation that established risk factors or other peripheral biomarkers can serve as common risk factors for both cerebrovascular and cognitive health. The current study sought to identify the relationship between several peripheral biomarkers and cognitive performance in older adults at increased risk of cognitive decline and dementia. The peripheral biomarkers [tumor necrosis factor- $\alpha$ (TNF- $\alpha$ ), vascular cell adhesion molecule-1 (VCAM-1), intracellular adhesion molecule-1 (ICAM-1), PAI-1 (plasminogen activator inhibitor-1), cluster of differentiation 40-ligand (CD40L)] have previously been demonstrated for their role in contributing to endothelial dysfunction and inflammation in cardiovascular disease $[8,9]$. Additionally, physical activity has been reported to influence the levels of brain-derived neurotrophic factor (BDNF) [10, 11], which has a role in neuronal survival [12], and there is conflicting data on its relationship to cognition [13-15]. Nonetheless, these biomarkers were chosen for the plausible mechanisms involving in endothelial dysfunction, neuroinflammation, or protective properties that may affect the neurovascular unit in the bloodbrain barrier interface. We hypothesized that both established cardiovascular risk factors and selected peripheral biomarkers would be associated with cognitive performance in older adults with memory complaints.

\section{MATERIALS AND METHODS}

\section{Study participants}

Participants in this study were selected from the Australian Imaging Biomarkers \& Lifestyle (AIBL) flagship study of ageing, following 1,112 older volunteers longitudinally from two sites in Melbourne and Perth. Participants in the AIBL Active study were selected from the AIBL cohort in light of their vascular risk only if they met the inclu- 
sion criteria: community-dwelling individuals over 60 years old from the Melbourne site, with MiniMental State Examination (MMSE) $>23$ and with at least one vascular risk factor [16]. The AIBL Active trial was designed to investigate whether a home-based physical activity program of 24 months delays the progression of white matter changes on MRI in older adults with memory complaints and mild cognitive impairment [17] (Trial registration: Australia New Zealand Clinical Trials Registry ACTRN 12611000612910). Participants selected for this analysis $(n=108)$ either had a subjective memory complaint without cognitive impairment on testing (SMC) or had mild cognitive impairment (MCI). Exclusion criteria included diagnosis of dementia with MMSE <24, depression with baseline Geriatric Depression Scale (GDS) >6 (254), and severe visual or hearing impairment [17]. All participants provided written informed consent. Ethics approval was obtained from the Melbourne Health Human Research Ethics Committee.

\section{Global cognitive tests}

The MMSE (score 0-30) [18] and the Alzheimer's disease Assessment Scale-Cognitive Section (ADAS-cog) (score range 0-70), incorporating a delayed verbal recall task, were used for analysis [19]. ADAS-cog consists of an 11-item battery of short neuropsychological tests. Both MMSE and ADAS-cog are common tools used to monitor cognitive performance in clinical trials. Lower MMSE and higher ADAS-cog scores indicate increasing severity of cognitive impairment. The Consortium to Establish a Registry for Alzheimer's Disease (CERAD) tool is sensitive to early cognitive impairment and was used to determine the presence or absence of MCI (defined as any CERAD subset equal or lower than $-1.5 \mathrm{SD}$ or more compared to the AIBL Active control group scores on any of the CERAD subtest) [20].

\section{Executive function and verbal memory tests}

Individual tests in the CERAD in verbal fluency and delayed recall were used to assess executive function and verbal memory respectively. Verbal fluency examines the contribution of verbal retrieval and executive control. It is a timed task that requires participants to provide as many words as possible, starting with a specified letter. Word list recall requires subjects to reproduce from memory lists of words after a 10 -s exposure (0 to 10 , a higher score indicating better function) [20].

All cognitive test scores were standardized due to a differing magnitude of variance according to the task. Trail Making Tests (TMT) A \& TMT B are two parts of a test of executive function that provides information about visual search, scanning, processing speed, and mental flexibility [21]. Trail A requires participants to draw lines sequentially connecting 25 encircled numbers. Similarly, Trail B involves drawing lines but alternates between numbers and letters.

The severity of affective symptoms was measured using the Hospital Anxiety and Depression Scale (HADS) [22]. HADS is a 14-item self-rating tool used to assess the presence and severity of anxiety and depressive symptoms in medical patients. The seven-item subscales for anxiety and depression are valid and reliable measures and are suitable to use in hospital outpatient, primary care, and community settings for all ages.

\section{Vascular risk factors}

A Framingham General 10-year Cardiovascular Risk Score was calculated using the office-based cardiovascular risk score algorithm derived from the Framingham Heart Study [23]. The Framingham risk score is one of the most widely used methods in the clinical setting to estimate the future risk of a cardiovascular event, which increases with a higher risk score [24]. It is recommended for use in the Australian primary care setting by Diabetes Australia, Kidney Health Australia, Heart Foundation and the Stroke Foundation [4]. The model takes into account age, sex, body mass index, blood pressure, smoking, and diabetes, with higher scores indicating an increased risk of cardiovascular events. We used self-reporting of cardiovascular health including smoking status, diagnosis and listing medications, and a physician researcher (ML) adjudicated the medical records. All participants provided written informed consent to access medical records from general practitioners and specialist cardiologists. This method of data collection is highly accurate with a low false negative rate of $2 \%$ [25].

\section{Blood analysis for established vascular risk factors and peripheral biomarkers}

Blood samples for analysis of established vascular risk factors were collected at baseline and sent to a certified medical laboratory (Melbourne Pathol- 
ogy, Melbourne, Australia) for determination of lipid concentrations (i.e., Total Triglycerides, LDLC, HDL-C, and Triglycerides), fasting insulin, plasma glucose, homocysteine, and hs-CRP. Additionally, plasma samples collected at baseline were screened in duplicate for a number of peripheral biomarkers known to be associated with vascular health and/or cognition such as TNF- $\alpha$, soluble ICAM-1 (sICAM-1), soluble VCAM-1 (sVCAM-1), PAI-1, monocyte chemoattractant protein-1 (MCP-1), soluble CD40L (sCD40L), and BDNF using Milliplex ${ }^{\circledR}$ MAP human disease panels (Millipore, Billerica, MA, USA) and analyzed using the Luminex ${ }^{\circledR}$ xMAP® technology with the Bio-Rad Bio-Plex System (Gladesville, NSW, Australia). The limit of detection for each biomarker ranged from 0.23 to $6.44 \mathrm{pg} / \mathrm{ml}$. For all biomarker assays, the intra-assay and inter-assay coefficients of variation were less than $10 \%$.

\section{Statistical analysis}

All analyses were conducted using SPSS (version 23) and STATA (version 11.2, StataCorp, TX, USA). The neuropsychological assessment consisted of tests to examine performance in related cognitive domains and, therefore, required a factor analysis of the cognitive domains. The scores of each neuropsychological test entered into an exploratory factor analysis to extract factors accounting for the pattern of correlations. The selection of oblimin rotation allowed factors to correlate. Preanalysis utilized the Kaiser-Meyer-Olkin (KMO) test of sampling adequacy (should be greater than 0.5) and Bartlett's test of sphericity ( $\mathrm{p}$ should be $<0.05$ ). A KMO test above 0.5 and $p<0.05$ in the Bartlett's test should indicate that the data were appropriate for exploratory factor analysis [26, 27]. Factor scores were used as cognitive outcomes for the regression analyses.

Covariates were identified a priori based on theoretical relevance with cognitive function and cardiovascular variables: age, sex, education, and depression [28, 29]. All regression models for the biomarkers were fit independently to identify any univariate association between cognitive domains (dependent variables) and the peripheral biomarker levels (independent variables). The association of each of the four cognitive domains (dependent variable) and established risk factors and peripheral biomarkers (independent variable) were assessed in four separate multivariable linear regression analyses adjusting for the covariates. The analysis was repeated separately for fasting glucose, LDLcholesterol, and Framingham scores. Regression diagnostics confirmed that the standard assumptions of the linear regression models were adequately met. There were 48 separate models (four response variables $\mathrm{x} 12$ predictors), and to adequately control for type I errors associated with multiple tests, we have generally highlighted only those results which were significant at $p<0.001(0.05 / 48>0.001)$. Other associations which were less significant have been given less weight.

A sample size of 108 would provide approximately $80 \%$ power to detect a two-point difference between groups with a significant level of 0.05 , based on an $\mathrm{SD}$ of 3.5 in ADAS-cog in the AIBL Active study.

\section{RESULTS}

Table 1 summarizes the characteristics of the participants. The mean age was 73 years, and $54 \%$ were female.

Table 1

Sample characteristics $(n=108)$

\begin{tabular}{lcc}
\hline Clinical characteristics & Mean & SD \\
\hline Age, y & 73.17 & 5.78 \\
Female, n (\%) & 58 & 53.7 \\
Education, y & 14.06 & 3.65 \\
Mild cognitive impairment, n (\%) & 32 & 29.6 \\
Subjective memory complaint, n $(\%)$ & 76 & 70.4 \\
HADS - depression & 2.98 & 2.52 \\
\hline Established VRF & & \\
\hline Fasting glucose (mmol/L) & 5.38 & 1.16 \\
Triglyceride (mmol/L) & 1.32 & 0.61 \\
Total cholesterol (mmol/L) & 5.18 & 1.09 \\
LDL-cholesterol (mmol/L) & 3.06 & 0.95 \\
HDL-cholesterol (mmol/L) & 1.52 & 0.39 \\
Framingham score & 10.02 & 7.12 \\
hs-CRP (mmol/L) & 2.22 & 3.18 \\
Homocysteine ( $\mu$ mol/L) & 9.44 & 3.46 \\
\hline Peripheral biomarkers & & \\
\hline sVCAM-1 (ng/ml) & 1,118 & 317 \\
sICAM-1 (ng/ml) & 128 & 689 \\
PAI-1 (ng/ml) & 74 & 37 \\
TNF- $\alpha$ (pg/ml) & 11.56 & 5.91 \\
BDNF (pg/ml) & 702.43 & 1163.24 \\
MCP-1 (pg/ml) & 256.11 & 90.87 \\
sCD40L (pg/ml) & 61.59 & 38.47 \\
\hline Val & & \\
\hline
\end{tabular}

Values are in mean (SD) or $\mathrm{n}(\%)$. VRF, vascular risk factor; TNF- $\alpha$, tumor necrosis factor-alpha; sVCAM-1, soluble vascular cell adhesion molecule-1; sICAM-1, soluble intercellular adhesion molecule-1; BDNF, brain-derived neurotrophic factor; MCP, monocyte chemotactic protein-1; PAI-1, plasminogen activating inhibitor-1; sCD40L, soluble CD40 ligand. 
Table 2

Cognitive domains and scores in neuropsychological testing

\begin{tabular}{llcc}
\hline Cognitive domains and skills & Tests & \multicolumn{2}{c}{ Raw scores $(n=108)$} \\
\cline { 3 - 4 } & & Mean & SD \\
\hline Global cognition & ADAS-cog & 7.56 & 3.81 \\
& MMSE & 28.65 & 1.60 \\
\hline Verbal memory & & & \\
\hline Immediate verbal memory & Immediate recall & 4.88 & 1.79 \\
Delayed verbal memory & Delayed recall & 6.95 & 2.05 \\
\hline Visuospatial skills/ psychomotor speed & & & \\
\hline Visual scanning, Psychomotor speed & Trail making test A & 40.20 & 13.15 \\
& Trail making test B & 70.98 & 61.61 \\
\hline Verbal fluency & & & \\
\hline Executive function & Verbal fluency & 20.33 & 4.34 \\
\hline
\end{tabular}

MMSE, Mini-Mental State Examination; ADAS-cog, Alzheimer's Disease Assessment Scale - cognitive subscale. The Trail Making Test A and B are measured in seconds.

Table 3

Separate linear regression models of individual vascular risk factor, peripheral biomarker, and cognitive performance

\begin{tabular}{lcccc}
\hline $\begin{array}{l}\text { Cognitive } \\
\text { domains }\end{array}$ & $\begin{array}{c}\text { Global } \\
\text { cognition }\end{array}$ & $\begin{array}{c}\text { Verbal } \\
\text { memory }\end{array}$ & $\begin{array}{c}\text { Verbal } \\
\text { fluency }\end{array}$ & $\begin{array}{c}\text { Visuospatial/ } \\
\text { psychomotor } \\
\text { speed }\end{array}$ \\
Tests & ADAS-cog & $\begin{array}{c}\text { CERAD delayed } \\
\text { recall }\end{array}$ & $\begin{array}{c}\text { CERAD verbal } \\
\text { fluency }\end{array}$ & $\begin{array}{c}\text { Trail making } \\
\text { test B }\end{array}$ \\
\hline Established vascular risk factors & & & & \\
\hline Fasting glucose & $0.25(0.09)$ & $-0.14(0.10)$ & $-0.03(0.10)$ & $0.40(0.10)$ \\
LDL-cholesterol & $-0.02(0.10)$ & $-0.04(0.10)$ & $0.07(0.10)$ & $-0.09(0.11)$ \\
Homocysteine & $0.23(0.10)$ & $-0.20(0.10)$ & $0.02(0.10)$ & $0.43(0.09)$ \\
Framingham score & $-0.12(0.19)$ & $-0.09(0.19)$ & $0.33(0.19)$ & $0.42(0.19)$ \\
hs-CRP & $-0.04(0.09)$ & $0.11(0.10)$ & $-0.04(0.10)$ & $-0.01(0.10)$ \\
\hline Novel biomarkers & & & & \\
\hline sVCAM-1 & $0.03(0.09)$ & $0.09(0.09)$ & $-0.06(0.10)$ & $0.05(0.10)$ \\
SICAM-1 & $-0.11(0.09)$ & $0.17(0.09)$ & $0.11(0.09)$ & $-0.06(0.10)$ \\
PAI-1 & $-0.03(0.09)$ & $0.01(0.10)$ & $-0.03(0.10)$ & $0.02(0.10)$ \\
TNF- $\alpha$ & $0.16(0.09)$ & $-0.05(0.10)$ & $-0.01(0.10)$ & $0.21(0.10)$ \\
BDNF & $-0.20(0.09)$ & $0.17(0.09)$ & $0.10(0.09)$ & $-0.07(0.10)$ \\
MCP & $-0.07(0.09)$ & $0.09(0.09)$ & $0.14(0.10)$ & $0.01(0.10)$ \\
sCD40L & $-0.16(0.09)$ & $0.13(0.09)$ & $-0.03(0.09)$ & $-0.03(0.10)$ \\
\hline Va & & & & \\
\hline
\end{tabular}

Values are standardized coefficient (SE). Separate linear regression models for vascular risk factors and peripheral biomarkers were adjusted for age, sex, education in years, and depression score (HADS-D). ADAS-cog, Alzheimer's Disease Assessment Scale - cognitive subscale; sVCAM-1, soluble vascular cell adhesion molecule-1; sICAM-1, soluble intercellular adhesion molecule-1; PAI-1, plasminogen activating inhibitor-1; TNF-alpha, tumor necrosis factor$\alpha$; BDNF, brain-derived neurotrophic factor; MCP, monocyte chemotactic protein-1; sCD40L, soluble CD40 ligand.

\section{Cognitive domains}

In factor analysis, the KMO test of sampling adequacy (0.57) and Barlett's test of sphericity $(p<0.001)$ indicated that the data were appropriate for exploratory factor analysis. Three factors were extracted: 1) verbal memory (CERAD word list immediate recall $86.4 \%$ and delayed recall $87 \%$ ), 2) verbal fluency (CERAD verbal fluency $98.1 \%$ ), and 3 ) visual-spatial/processing speed (TMT A $82.6 \%$ and TMT B $86.2 \%$ ) (Table 2). The three components explained $80.2 \%$ of the variance of the original data.

\section{Vascular risk factors}

Table 3 shows the relationship between vascular risk factors, peripheral biomarkers. and cognitive performance. Performance in verbal fluency decreased as age increased. Higher fasting glucose was a factor associated with an increase in psychomotor speed using TMT B (adjusted $\beta=0.40 \pm 0.10, p<0.001$ ), and there was some evidence of it being associated with higher scores using ADAS-cog, reflecting poorer global cognition (adjusted $\beta=0.25 \pm 0.09, p<0.01$ ). Higher 
homocysteine levels were associated with poorer performance in TMT B (adjusted $\beta=0.43 \pm 0.09$, $p<0.001$ ), and more weakly associated with poorer performance in global cognition using ADAS-cog (adjusted $\beta=0.23 \pm 0.10, p<0.05$ ), and delayed recall (adjusted $\beta=-0.20 \pm 0.10, p<0.05$ ).

There was some evidence of increasing Framingham scores being associated with poorer performance in TMT B (adjusted standardized $\beta=0.42 \pm 0.19$, $p<0.05)$. LDL-cholesterol and hs-CRP almost showed no association with cognitive performance.

\section{Peripheral biomarkers}

Possible associations were evident between increasing TNF- $\alpha$ and lower performance in TMT B (adjusted standardized $\beta=0.21 \pm 0.10$, $p<0.05)$ and between increasing BDNF and better global cognitive performance (adjusted standardized $\beta=-0.20 \pm 0.09, p<0.05$ ), after controlling for age, sex, education, and depressive symptoms. MCP-1 and SCD40L showed almost no association with any cognitive domains.

After controlling for age, sex, education, and depressive symptoms, sVCAM-1, sICAM-1, and PAI showed almost no relationship with cognitive performance.

\section{DISCUSSION}

This study confirmed the positive linear association between increasing fasting glucose, homocysteine levels, and decreased performance in executive dysfunction as reported elsewhere [30]. The relationships remain significant after adjustment for age, sex, education, and depressive symptoms. The findings in the peripheral biomarker analyses add to the early evidence of associations between TNF- $\alpha$, BDNF, and cognitive deficits, which are not well established in the current cognitive literature.

\section{Established vascular risk factors}

Diabetes and impaired fasting glucose increase the risk of dementia and cognitive impairment [31]. However, the relationship between preceding stages of diabetes, including high normal blood glucose and cognitive pattern has not been fully demonstrated [31]. Our study found that higher glucose levels are associated with poorer global cognition and TMT $\mathrm{B}$, a sensitive test for executive functioning. One possible explanation may involve lower grey/white matter regional volumes in the frontal cortices, which are associated with higher blood glucose levels in the normal range in a study of people in late life [31]. Other factors impacting on cognitive performance may include hyperglycemia, hypoglycemia, and insulin deficiency and resistance observed in established diabetes [32].

This study demonstrated associations between homocysteine levels and cognitive performance in three cognitive tests. The association was strongest in the TMT B, requiring executive function with multiple skills such as visual screening ability and psychomotor processing speed. This observation is consistent with previous studies [33], suggesting that homocysteine may be a risk factor for cognitive deficits. Elevated homocysteine is a strong risk factor for cardiovascular disease and stroke. A $25 \%$ lower homocysteine level was found to show an associated 11\% lower IHD risk and 19\% lower stroke risk (homocysteine studies collaboration) [34]. Researchers have also reported an association between elevated homocysteine levels and an increased incidence of $\mathrm{AD}$ and vascular dementia [35].

The Framingham algorithm provides calculated risks based on blood test results, age, sex, and smoking status and inform whether an individual has optimal control of the modifiable risk factors in the primary prevention of cardiovascular disease and the risk scores predict stroke risk and cognitive performance $[29,36]$. The use of the Framingham algorithm has identified people with cerebral atrophy and inefficiencies in executive function using TMT A, TMT $B$, and visual-spatial function [30]. Similar to these results, the current study showed its relationship with TMT B but did not find changes in verbal memory [29]. As the Framingham scores consistently correlate with cognitive deficits in similar domains, our findings supported the appropriate use of Framingham scores as a collective measure to capture the total burden of vascular disease of the brain [1]. Furthermore, only four participants were smokers $(3.7 \%)$; therefore, the smoking status did not affect the results materially in our study population.

\section{Peripheral biomarkers}

Inflammation is a key component in $\mathrm{AD}$ and cerebrovascular diseases [37, 38]. TNF- $\alpha$ is an inflammatory cytokine upstream of IL-1, which has a potential role in the hippocampus after surgery. Animal models also support that this inflammatory 
marker may mediate through inducing upregulation of adhesion molecules such as SVCAM-1 [39]. Our result indicated that TNF- $\alpha$ had a possible linear relationship with TMT B (adjusted standardized $\beta=0.21 \pm 0.02, p<0.05$ ), indicating that such inflammation can play a role in cognitive deficit and is consistent with the notion that TNF- $\alpha$ may be a marker of future risk of developing AD [40].

BDNF, a growth factor member of the neurotrophin family, plays a pivotal role in neuronal survival and synaptic plasticity [12]. The growth factor has antidiabetic properties with low levels in type 2 diabetes mellitus and an inverse correlation with plasma glucose and insulin resistance. Reduced serum BDNF levels associated with cognitive deficits in diabetic patients [41]. The marker is also important for learning and memory by long-term potentiation in the hippocampus with structural changes in synapses. In a genetic polymorphism study in preclinical $A D$ using samples obtained from participants in the AIBL flagship study, allelic variation in BDNF Val66Met accelerated amyloid- $\beta$ (A $\beta$ ) related neurodegeneration with decline in hippocampal volume and episodic memory loss [42]. While further analysis did not demonstrate a relationship between serum BDNF with episodic memory [43], there was some evidence in this current study that low BDNF levels were related to reduced global cognitive performance on the ADAS-cog. This relationship is consistent with another study in which BDNF levels in the brain were reduced in patients with MCI and had a positive correlation with cognitive performance [44, 45]. Furthermore, lower serum levels were observed in patients with stroke, vascular dementia and late-onset AD comparing with controls [12]

Upregulation of sVCAM-1 and sICAM-1 can reduce the permeability of small vessels through interaction with leucocytes [39], therefore, their high levels may serve as a marker for endothelial activation or dysfunction in the pathogenesis of atherosclerosis. Increased plasma ICAM-1 can be present in patients with coronary heart disease. Its association with white matter lesion suggests underlying inflammatory processes in large and small vessel diseases [46]. Plasma VCAM-1 levels were higher in patients with $\mathrm{AD}$ and vascular dementia than controls, indicating existence of endothelial dysfunction in both types of dementia [47], but did not predict new onset of dementia in a cohort of 727 healthy adults In the Rotterdam study [48]. It is possible that our study sample including 32 people with MCI and 76 with SMC has limited power to detect a small association.
The strength of the current biomarker study involves the use of comprehensive cognitive tests, in contrast to many biomarker studies that have examined cognitive performance using a single screening test, specifically MMSE with inadequate sensitivity, or using limited batteries that have not assessed cognitive function broadly [33]. Our factor analysis supports the extraction of the specific components to characterize the cognitive profile in our population. Such factors are consistent with the cognitive domains identified as vulnerable in other studies of vascular cognitive impairment [30].

Our study sample included older adults with SMC and MCI and may not be generalized to people with no memory complaints or people living with dementia. Because the AIBL study excluded volunteers with history or neuroimaging evidence of strokes, our findings could not be generalized in older adults who perform poorly on cognitive tests due to stroke. The observational data allowed cross-sectional examination of the relationship between vascular risk factors and cognitive performance, hence, does not imply causative relationships. The Framingham scores have some limitations. Cardiovascular factors captured in the Framingham risk scores included the non-clinical factor of smoking behavior, but the smoking rate is very low $(n=4)$ among our participants. Therefore, adding this behavioral factor to the scores may not be particularly useful in our study population. Furthermore, Framingham risk scores do not account for the effects of central obesity in the algorithm, but overweight and obesity was prevalent (69.4\%) in the study population.

Our study identified that established vascular risk factors (fasting glucose levels, Framingham scores, homocysteine) are associated with cognitive performance in various domains. These findings together with the potential association with the peripheral biomarker (TNF- $\alpha$ and BDNF) offer new insights and future directions for research into the molecular mechanisms in vascular cognitive impairment. Adequately powered longitudinal studies are required to examine their causal relationship and possible use in risk stratification or future clinical value in identifying individuals at risk of cognitive decline.

\section{ACKNOWLEDGMENTS}

We thank the Australian Imaging Biomarker and Lifestyle Study of Ageing participants for their participation, the participating research staff at 
the National Aging Research Institute (NARI) and the Mental Health Research Institute (MHRI) for the collection of physical assessments and the administration of the study.

This work is supported by a project grant from the Australian National Health and Medical Research Council awarded to NL, PMD, KC, DA, KE, MJS, and CM (ID: 1005492).

Authors' disclosures available online (https:// www.j-alz.com/manuscript-disclosures/19-0953r2).

\section{REFERENCES}

[1] Selnes OA, Vinters HV (2006) Vascular cognitive impairment. Nat Clin Pract Neurol 2, 538-547.

[2] Chertkow H, Feldman HH, Jacova C, Massoud F (2013) Definitions of dementia and predementia states in Alzheimer's disease and vascular cognitive impairment: Consensus from the Canadian conference on diagnosis of dementia. Alzheimers Res Ther 5, S2.

[3] Obeid R, Herrmann W (2006) Mechanisms of homocysteine neurotoxicity in neurodegenerative diseases with special reference to dementia. FEBS Lett 580, 2994-3005.

[4] National Vascular Disease Prevention Alliance (2009) Guidelines for the Assessment of Absolute Cardiovascular Disease Risk.

[5] Whitmer RA, Sidney S, Selby J, Johnston SC, Yaffe K (2005) Midlife cardiovascular risk factors and risk of dementia in late life. Neurology 64, 277-281.

[6] Gorelick PB, Scuteri A, Black SE, Decarli C, Greenberg SM, Iadecola C, Launer LJ, Laurent S, Lopez OL, Nyenhuis D, Petersen RC, Schneider JA, Tzourio C, Arnett DK, Bennett DA, Chui HC, Higashida RT, Lindquist R, Nilsson PM, Roman GC, Sellke FW, Seshadri S, American Heart Association Stroke Council, Council on Epidemiology and Prevention, Council on Cardiovascular Nursing, Council on Cardiovascular Radiology and Intervention, and Council on Cardiovascular Surgery and Anesthesia (2011) Vascular contributions to cognitive impairment and dementia: A statement for healthcare professionals from the American Heart Association/American Stroke Association. Stroke 42, 2672-2713.

[7] Kruman II, Culmsee C, Chan SL, Kruman Y, Guo Z, Penix L, Mattson MP (2000) Homocysteine elicits a DNA damage response in neurons that promotes apoptosis and hypersensitivity to excitotoxicity. J Neurosci 20, 6920-6926.

[8] Wang J, Tan GJ, Han LN, Bai YY, He M, Liu HB (2017) Novel biomarkers for cardiovascular risk prediction. J Geriatr Cardiol 14, 135-150.

[9] Schondorf T, Lubben G, Karagiannis E, Erdmann E, Forst T, Pfutzner A (2010) Increased prevalence of cardiovascular disease and risk biomarkers in patients with unknown type 2 diabetes visiting cardiology specialists: Results from the DIASPORA study. Diab Vasc Dis Res 7, 145-150.

[10] Coelho FG, Vital TM, Stein AM, Arantes FJ, Rueda AV, Camarini R, Teodorov E, Santos-Galduroz RF (2014) Acute aerobic exercise increases brain-derived neurotrophic factor levels in elderly with Alzheimer's disease. J Alzheimers Dis 39, 401-408.

[11] Gregoire CA, Berryman N, St-Onge F, Vu TTM, Bosquet L, Arbour N, Bherer L (2019) Gross motor skills training leads to increased brain-derived neurotrophic factor levels in healthy older adults: A pilot study. Front Physiol 10,410.

[12] Passaro A, Dalla Nora E, Morieri ML, Soavi C, Sanz JM, Zurlo A, Fellin R, Zuliani G (2015) Brain-derived neurotrophic factor plasma levels: Relationship with dementia and diabetes in the elderly population. J Gerontol A Biol Sci Med Sci 70, 294-302.

[13] Dias VV, Brissos S, Frey BN, Andreazza AC, Cardoso C, Kapczinski F (2009) Cognitive function and serum levels of brain-derived neurotrophic factor in patients with bipolar disorder. Bipolar Disord 11, 663-671.

[14] Dols A, Thesing CS, Bouckaert F, Oude Voshaar RC, Comijs HC, Stek ML (2015) BDNF serum levels are not related to cognitive functioning in older depressed patients and controls. Int Psychogeriatr 27, 649-656.

[15] De la Rosa A, Solana E, Corpas R, Bartres-Faz D, Pallas M, Vina J, Sanfeliu C, Gomez-Cabrera MC (2019) Long-term exercise training improves memory in middle-aged men and modulates peripheral levels of BDNF and Cathepsin B. Sci Rep 9, 3337.

[16] Ellis KA, Bush AI, Darby D, De Fazio D, Foster J, Hudson P, Lautenschlager NT, Lenzo N, Martins RN, Maruff P, Masters C, Milner A, Pike K, Rowe C, Savage G, Szoeke C, Taddei K, Villemagne V, Woodward M, Ames D, AIBL Research Group (2009) The Australian Imaging, Biomarkers and Lifestyle (AIBL) study of aging: Methodology and baseline characteristics of 1112 individuals recruited for a longitudinal study of Alzheimer's disease. Int Psychogeriatr 21, 672-687.

[17] Cyarto EV, Lautenschlager NT, Desmond PM, Ames D, Szoeke C, Salvado O, Sharman MJ, Ellis KA, Phal PM, Masters CL, Rowe CC, Martins RN, Cox KL (2012) Protocol for a randomized controlled trial evaluating the effect of physical activity on delaying the progression of white matter changes on MRI in older adults with memory complaints and mild cognitive impairment: The AIBL Active trial. BMC Psychiatry 12, 167.

[18] Folstein MF, Folstein SE, McHugh PR (1975) "Minimental state". A practical method for grading the cognitive state of patients for the clinician. J Psychiatr Res 12, 189-198.

[19] Rosen WG, Mohs RC, Davis KL (1984) A new rating scale for Alzheimer's disease. Am J Psychiatry 141, 1356-1364.

[20] Welsh KA, Butters N, Mohs RC, Beekly D, Edland S, Fillenbaum G, Heyman A (1994) The Consortium to Establish a Registry for Alzheimer's Disease (CERAD). Part V. A normative study of the neuropsychological battery. Neurology 44, 609-614.

[21] Reitan R, Wolfson D (1985) The Halstead-Reitan Neuropsychological Test Battery, Neuropsychology Press, Tuscan.

[22] Zigmond AS, Snaith RP (1983) The hospital anxiety and depression scale. Acta Psychiatr Scand 67, 361-370.

[23] Framingham risk calculator, http://cvdrisk.nhlbi.nih.gov.

[24] Kuklina EV (2010) Assessing and managing risk for cardiovascular disease: A worldwide perspective. $N$ Am J Med Sci (Boston) 3, 94-103.

[25] Barr EL, Tonkin AM, Welborn TA, Shaw JE (2009) Validity of self-reported cardiovascular disease events in comparison to medical record adjudication and a statewide hospital morbidity database: The AusDiab study. Intern Med J 39, 49-53.

[26] Kaiser HF (1974) An index of factorial simplicity. Psychometrika 39, 31-36.

[27] Bartlett MS (1951) The effect of standardization on a chi square approximation in factor analysis. Biometrika $\mathbf{3 8}$, 337-344. 
[28] Reijmer YD, van den Berg E, Dekker JM, Nijpels G, Stehouwer CD, Kappelle LJ, Biessels GJ (2011) The metabolic syndrome, atherosclerosis and cognitive functioning in a non-demented population: The Hoorn Study. Atherosclerosis 219, 839-845.

[29] Kaffashian S, Dugravot A, Elbaz A, Shipley MJ, Sabia S, Kivimaki M, Singh-Manoux A (2013) Predicting cognitive decline: A dementia risk score vs. the Framingham vascular risk scores. Neurology 80, 1300-1306.

[30] Seshadri S, Wolf PA, Beiser A, Elias MF, Au R, Kase CS, D'Agostino RB, DeCarli C (2004) Stroke risk profile, brain volume, and cognitive function: The Framingham Offspring Study. Neurology 63, 1591-1599.

[31] Mortby ME, Janke AL, Anstey KJ, Sachdev PS, Cherbuin N (2013) High "normal" blood glucose is associated with decreased brain volume and cognitive performance in the 60s: The PATH through life study. PLoS One 8, e73697.

[32] Kawamura T, Umemura T, Hotta N (2012) Cognitive impairment in diabetic patients: Can diabetic control prevent cognitive decline? J Diabetes Investig 3, 413-423.

[33] Schafer JH, Glass TA, Bolla KI, Mintz M, Jedlicka AE, Schwartz BS (2005) Homocysteine and cognitive function in a population-based study of older adults. $J$ Am Geriatr Soc 53, 381-388.

[34] Homocysteine Studies C (2002) Homocysteine and risk of ischemic heart disease and stroke: A meta-analysis. JAMA 288, 2015-2022.

[35] Prins ND, Den Heijer T, Hofman A, Koudstaal PJ, Jolles J, Clarke R, Breteler MM, Rotterdam Scan S (2002) Homocysteine and cognitive function in the elderly: The Rotterdam Scan Study. Neurology 59, 1375-1380.

[36] Elias MF, Sullivan LM, D'Agostino RB, Elias PK, Beiser A, Au R, Seshadri S, DeCarli C, Wolf PA (2004) Framingham stroke risk profile and lowered cognitive performance. Stroke 35, 404-409.

[37] Weinstein G, Seshadri S (2014) Circulating biomarkers that predict incident dementia. Alzheimers Res Ther 6, 6.

[38] Sharman MJ, Verdile G, Kirubakaran S, Parenti C, Singh A, Watt G, Karl T, Chang D, Li CG, Munch G (2019) Targeting inflammatory pathways in Alzheimer's disease: A focus on natural products and phytomedicines. CNS Drugs 33, 457480.

[39] Ewers M, Mielke MM, Hampel H (2010) Blood-based biomarkers of microvascular pathology in Alzheimer's disease. Exp Gerontol 45, 75-79.
[40] Tan ZS, Beiser AS, Vasan RS, Roubenoff R, Dinarello CA, Harris TB, Benjamin EJ, Au R, Kiel DP, Wolf PA, Seshadri S (2007) Inflammatory markers and the risk of Alzheimer disease: The Framingham Study. Neurology 68, 1902-1908.

[41] Zhen YF, Zhang J, Liu XY, Fang H, Tian LB, Zhou DH, Kosten TR, Zhang XY (2013) Low BDNF is associated with cognitive deficits in patients with type 2 diabetes. Psychopharmacology (Berl) 227, 93-100.

[42] Lim YY, Hassenstab J, Cruchaga C, Goate A, Fagan AM, Benzinger TL, Maruff P, Snyder PJ, Masters CL, Allegri R, Chhatwal J, Farlow MR, Graff-Radford NR, Laske C, Levin J, McDade E, Ringman JM, Rossor M, Salloway S, Schofield PR, Holtzman DM, Morris JC, Bateman RJ, Dominantly Inherited Alzheimer Network (2016) BDNF Val66Met moderates memory impairment, hippocampal function and tau in preclinical autosomal dominant Alzheimer's disease. Brain 139, 2766-2777.

[43] Lim YY, Rainey-Smith S, Lim Y, Laws SM, Gupta V, Porter T, Bourgeat P, Ames D, Fowler C, Salvado O, Villemagne VL, Rowe CC, Masters CL, Zhou XF, Martins RN, Maruff P (2017) BDNF Val66Met in preclinical Alzheimer's disease is associated with short-term changes in episodic memory and hippocampal volume but not serum mBDNF. Int Psychogeriatr 29, 1825-1834.

[44] Peng S, Wuu J, Mufson EJ, Fahnestock M (2005) Precursor form of brain-derived neurotrophic factor and mature brainderived neurotrophic factor are decreased in the pre-clinical stages of Alzheimer's disease. J Neurochem 93, 1412-1421.

[45] Connor B, Young D, Yan Q, Faull RL, Synek B, Dragunow M (1997) Brain-derived neurotrophic factor is reduced in Alzheimer's disease. Brain Res Mol Brain Res 49, 71-81.

[46] Han JH, Wong KS, Wang YY, Fu JH, Ding D, Hong Z (2009) Plasma level of sICAM-1 is associated with the extent of white matter lesion among asymptomatic elderly subjects. Clin Neurol Neurosurg 111, 847-851.

[47] Zuliani G, Cavalieri M, Galvani M, Passaro A, Munari MR, Bosi C, Zurlo A, Fellin R (2008) Markers of endothelial dysfunction in older subjects with late onset Alzheimer's disease or vascular dementia. J Neurol Sci 272, 164-170.

[48] Engelhart MJ, Geerlings MI, Meijer J, Kiliaan A, Ruitenberg A, van Swieten JC, Stijnen T, Hofman A, Witteman JC, Breteler MM (2004) Inflammatory proteins in plasma and the risk of dementia: The rotterdam study. Arch Neurol 61, 668-672. 